\title{
Thoracic Wall Reconstruction with Acellular Porcine Dermal Collagen Matrix
}

\author{
Joachim Schmidt ${ }^{1}$ Bassam Redwan ${ }^{1}$ Volkan Koesek ${ }^{1}$ Barbara Heitplatz ${ }^{2}$ Benedetta Bedetti ${ }^{1}$ \\ Hermann Aebert ${ }^{1}$ Karsten Wiebe ${ }^{1}$
}

${ }^{1}$ Department of Cardiothoracic Surgery, Division of Thoracic Surgery and Lung Transplantation, University Hospital of Muenster, Muenster, Germany

${ }^{2}$ Gerhard Domagk Institute of Pathology, University Hospital of Muenster, Muenster, Germany

Address for correspondence Joachim Schmidt, MD, Department of Cardiothoracic Surgery, University Hospital of Muenster, AlbertSchweitzer Campus Building A1, Muenster 48149, Germany (e-mail: joachim.schmidt@ukmuenster.de).

Thorac Cardiovasc Surg 2016;64:245-251.

\begin{abstract}
Background Major thoracic wall resections require the implantation of foreign materials for reconstruction and stabilization. Recently, biological collagen matrixes have emerged as an alternative to the routinely used synthetic materials.

Materials and Methods Retrospectively, we analyzed our initial experience of chest wall reconstruction on large defects using a cross-linked porcine dermal acellular collagen matrix mesh with a thickness of $1.5 \mathrm{~mm}$.

Results Six sarcoma patients with a mean age of 46 (22-66) years underwent chest wall resections. Complete thoracic wall defects (mean area $149 \mathrm{~cm}^{2}$ ) ranged from $8 \times 10$ to $15 \times 20 \mathrm{~cm}$ in size. In the majority of cases, only mobilized subcutaneous tissue and skin were used for soft-tissue coverage of the implanted porcine collagen matrix patches. Implantation and postoperative courses were uneventful in all patients. No local infections or wound healing problems occurred. The collagen material resulted in durable and good to excellent chest wall stability in clinical follow-ups, and on

Keywords

- chest wall

- sarcoma

- wound healing

- sternum

- tumor computed tomography scans spanning over 3.5 years. Histological examination showed integration, neovascularization, and long-term persistence of the collagen matrix on late reoperation of one patient.

Conclusion Acellular porcine dermal collagen matrix is a feasible and reliable biological patch material for reconstruction of the thoracic wall. Excellent wound healing and long-term stability are achieved even in large defects or complete sternal replacements.
\end{abstract}

\section{Introduction}

Chest wall resections due to malignancies require extended surgical procedures to ensure wide safety margins. Reconstruction of larger defects, and in particular complete sternal resections, presents a major challenge. ${ }^{1}$ Sufficient chest wall stability, absence of respiratory impairments, adequate tissue coverage, and satisfactory cosmetics results must all be achieved. $^{2}$

received

February 23, 2014

accepted after revision

April 28, 2014

published online

July 28, 2014

Synthetic materials combined with soft-tissue transfers, such as pectoralis or latissimus muscle flaps, have been employed in chest wall reconstructions for decades. ${ }^{2,3}$ This strategy has been associated with significant morbidity and mortality in 46 to $69 \%$ of patients. ${ }^{4,5}$ Respiratory impairments may pose major problems such as paradox chest wall movements and rigidity due to excessive encapsulation. ${ }^{6}$ Seroma formation and wound healing impairment are paving the way for infection of synthetic materials which

(c) 2016 Georg Thieme Verlag KG Stuttgart · New York
DOI http://dx.doi.org/ 10.1055/s-0034-1383512. ISSN 0171-6425. 
Table 1 Patient characteristics

\begin{tabular}{|l|l|l|l|l|l|l|}
\hline Patient & $\begin{array}{l}\text { Sex } \\
(\mathrm{M} / \mathrm{F})\end{array}$ & Age (y) & Diagnosis & Location & Defect size $\left.\mathbf{( c m}^{2}\right)$ & Pretreatment \\
\hline 1 & $\mathrm{M}$ & 22 & Osteosarcoma (G3) & Ninth rib left & $9 \times 18 \mathrm{~cm} \mathrm{(162)}$ & $\begin{array}{l}\text { Preoperative } \\
\text { chemotherapy }\end{array}$ \\
\hline 2 & $\mathrm{~F}$ & 48 & High-grade sarcoma (G3) & $\begin{array}{l}\text { Dorsal chest } \\
\text { wall left (ribs 7-9) }\end{array}$ & $8 \times 10 \mathrm{~cm} \mathrm{(80)}$ & None \\
\hline 3 & $\mathrm{M}$ & 44 & Chondrosarcoma (G2) & Sternum & $7 \times 19 \mathrm{~cm} \mathrm{(133)}$ & None \\
\hline 4 & $\mathrm{~F}$ & 50 & Ewing sarcoma (G4) & Seventh rib right & $14 \times 10 \mathrm{~cm} \mathrm{(140)}$ & $\begin{array}{l}\text { Preoperative } \\
\text { chemotherapy }\end{array}$ \\
\hline 5 & $\mathrm{M}$ & 66 & $\begin{array}{l}\text { Metastasis from synovial } \\
\text { sarcoma (G3) of the } \\
\text { right femur }\end{array}$ & $\begin{array}{l}\text { Chest wall } \\
\text { right (ribs 4-6) }\end{array}$ & $14 \times 18 \mathrm{~cm} \mathrm{(252)}$ & $\begin{array}{l}\text { Preoperative } \\
\text { chemotherapy }\end{array}$ \\
\hline 6 & $\mathrm{~F}$ & 46 & Chondrosarcoma (G2) & Sternum & $8 \times 16 \mathrm{~cm} \mathrm{(128)}$ & None \\
\hline
\end{tabular}

often jeopardizes long-term success and result in significant failure rates. ${ }^{7}$

In the past, the use of a wide variety of synthetic materials such as polypropylene, polytetrafluoroethylene (PTFE), solid methylmethacrylate sandwich constructions, as well as various composite absorbable meshes has been explored. PTFE materials have been most frequently used. ${ }^{8}$ However, due to the inconclusiveness of available studies, the type of allomaterial and the technique of reconstruction still depend largely on the expertise and preference of the individual surgeon.

Recently, collagen matrixes harvested from human or animal sources and processed for medical use have emerged as an alternative. The implanted matrix undergoes a remodeling process with cellular infiltration, neovascularization, and exchange of extracellular matrix. Some of these materials, such as the product used in the present study, have been chemically cross-linked to increase strength and durability. ${ }^{9-13}$

The implantation of biological collagen matrixes has been described in abdominal hernia repair and various other fields of surgery. ${ }^{11,12,14}$ However, in thoracic surgery, experience is limited to single cases and small series. ${ }^{15-17}$ Unlike permanently fixated biological and synthetic materials, acellular collagen matrixes allow for integration and remodeling. The long-term integrity, strength, and stability of a collagen matrix are unknown. In this pilot study, we evaluated the feasibility and long-term results in the usage of a porcine dermal collagen matrix for thoracic wall reconstruction.

\section{Materials and Methods}

\section{Study Setting and Design}

Beginning in December 2009, six consecutive sarcoma patients scheduled for major thoracic wall or sternum resection were enrolled in the study (-Table 1). Written informed consent was obtained from all patients. Data were analyzed retrospectively and included patient demographics, diagnoses, operative data, and both pre- and postoperative laboratory and technical examinations. All patients underwent preoperative and postoperative computed tomography (CT) scans required for tumor evaluation and oncological follow-up.

\section{Characteristics of Biological Implant}

Thoracic wall reconstructions were performed using collagen matrix patches (Permacol; Covidien, Mansfield, Massachusetts, United States; $1.5 \mathrm{~mm}$ thickness in sizes of up to $15 \times 20 \mathrm{~cm})$ (-Figs. 1 and $\mathbf{2}$ ). This collagen matrix is derived
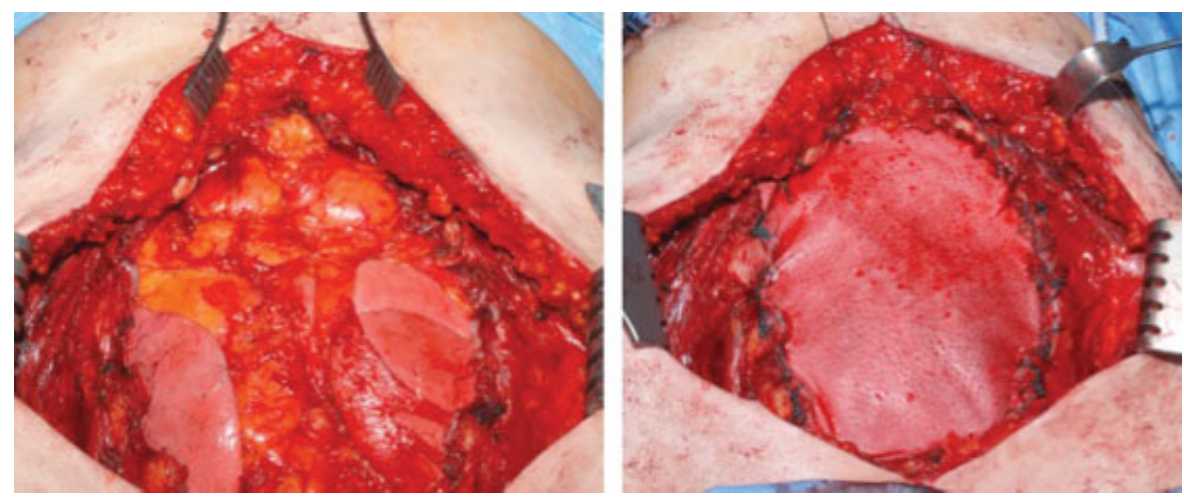

Fig. 1 Complete sternum resection in a patient with chondrosarcoma (left panel). Subsequent sternum reconstruction with a $15 \times 20 \mathrm{~cm}$ porcine dermal derived patch (right panel). 


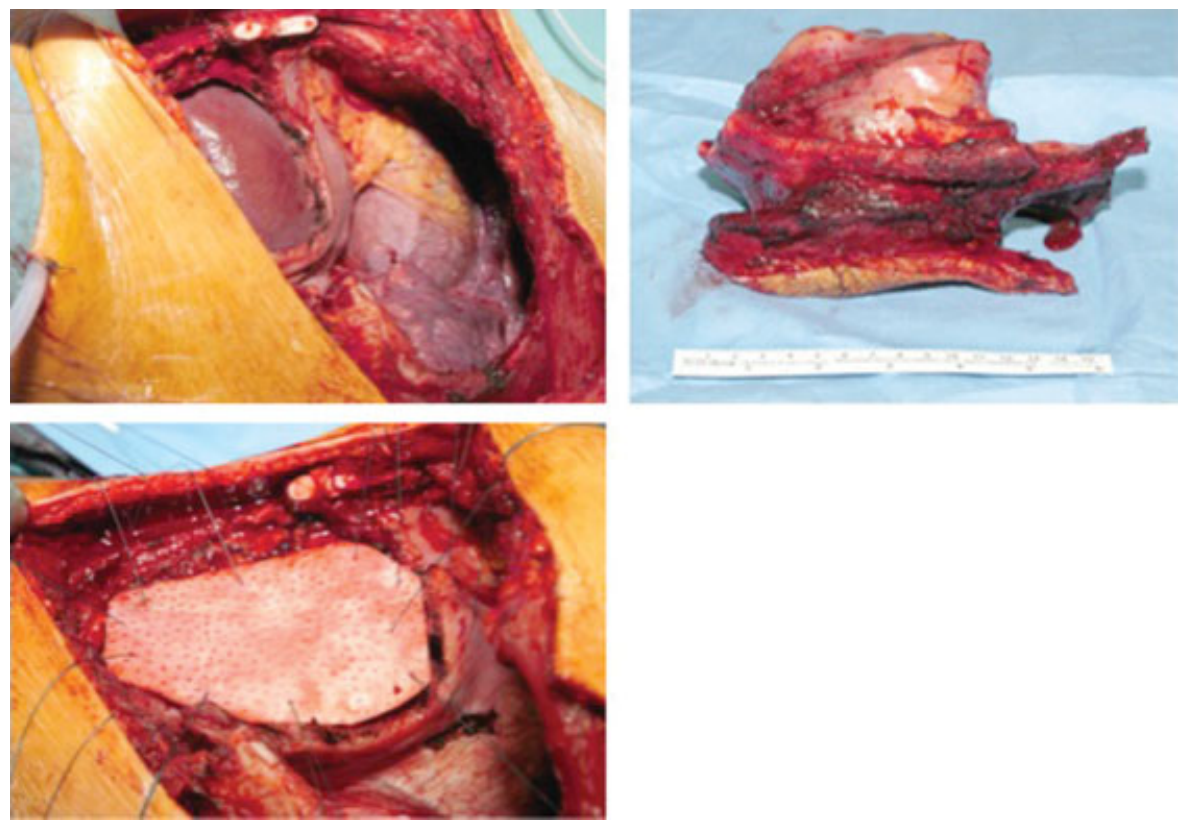

Fig. 2 Extended thoracic wall resection in a patient with osteosarcoma of the ninth left rib (upper panels). Partial diaphragm resection and pulmonary wedge resections were necessary. The chest wall and diaphragm defects were reconstructed using a $15 \times 20 \mathrm{~cm}$ Permacol (Covidien, Mansfield, Massachusetts, United States) patches (lower panel). A pedicled latissimus dorsi transfer flap was used for soft-tissue coverage.

from porcine dermis in which cells, cell debris, DNA, and RNA were removed. The resulting acellular matrix with its constituent collagen fibers is cross-linked with hexamethylene diisocyanate for additional stability and reduction of collagenase degradation. The biomechanical characteristics of several collagen materials have been tested in experimental settings. The native collagen patch used in this series sustained a maximum load of $317.2 \pm 23.6 \mathrm{~N}$ during uniaxial tensile testing. The tensile strength per unit width was $105.7 \pm 7.9 \mathrm{~N} / \mathrm{cm}$ and stiffness $58.3 \pm 4.0 \mathrm{~N} / \mathrm{mm}$ (mean \pm standard error of the mean). One month after abdominal hernia repair in a porcine animal model, the maximum tolerated force was $35.6 \pm 3.5 \mathrm{~N}$ compared with the native abdominal wall with $16.8 \pm 1.8 \mathrm{~N}$, similarly the tensile strength was $11.9 \pm 1.2 \mathrm{~N} / \mathrm{cm}$ compared with $5.6 \pm 0.6 \mathrm{~N} /$ $\mathrm{cm}$ and stiffness values were $5.8 \pm 0.9 \mathrm{~N} / \mathrm{mm}$ compared with $1.1 \pm 0.2 \mathrm{~N} / \mathrm{mm}^{10-12,14,18,19}$

\section{Patients and Surgical Techniques}

Indications for surgery were resection of primary chest wall sarcomas $(n=5)$ or sarcoma metastasis $(n=1)$. Patient data are listed in - Table 1 . Lateral chest wall resections were performed via thoracotomies to ensure wide safety margins $(n=4)$. Complete sternal resections were performed through a midline incision (-Figs. 1 and $\mathbf{2}$ ).

Before treatment, all patients had undergone biopsy to determine definite histopathological diagnosis. All tumor surgeries were en bloc resections, which included the biopsy sites.

Resections were performed with a minimum margin of $4 \mathrm{~cm}$ for ribs, sternum, and soft tissue. The underlying pleura was always resected, as were adherent parts of the lung, diaphragm, and mediastinum (-Table $\mathbf{1}$ ).
Thoracic cavities were drained with 28 Charrière chest tubes. Patches were shaped with scissors to match the resected defect with an overlap of approximately $1 \mathrm{~cm}$ on all margins to ensure complete coverage. Under tension the patch was anchored with multiple single-tied coated nonabsorbable polyethylene terephthalate sutures (Ethibond; Ethicon, Norderstedt, Germany) to neighboring ribs and transosseously drilled holes in the adjoining rib stumps. The same sutures were additionally used as running stiches ( - Fig. 3) to increase tension and to approximate soft tissues to the patch.

Redon drainages were placed on top, and left in place for at least 7 days. Adjacent muscles, subcutaneous tissue, and skin were mobilized, and approximated with polyglactin (Vicryl, Ethicon, Norderstedt, Germany) sutures. In two cases, soft-tissue coverage was augmented by transposition of lifted muscle flaps.

Before incision, a single shot of cefuroxime was administered for perioperative antibiotic prophylaxis. All patients received continuous postoperative analgesia via a peridural catheter. Patients were mobilized without restrictions from postoperative day 1 . Clinical wound assessment, laboratory tests, and radiological imaging were performed according to routine surgical procedures.

\section{Follow-Up}

Chest wall reconstructions were evaluated during postoperative oncological follow-up visits at 3, 6, 12, and 24 months. The subjective impressions or complaints from the patients were documented. Wound healing and soft-tissue coverage were examined. Chest wall stability was tested using deep breath maneuvers and coughing tests. The stability was classified as excellent, good, medium, or poor according to the surgical consultant's impression. Routine oncological CT 

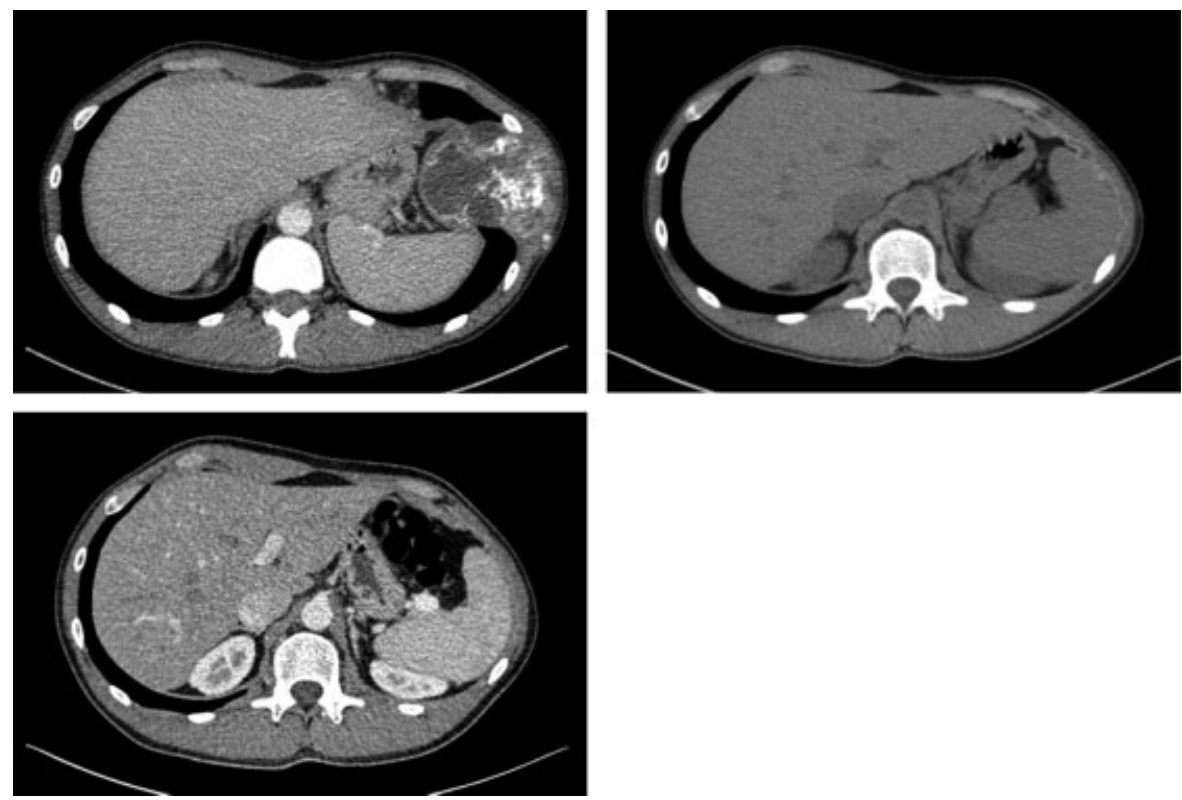

Fig. 3 Computed tomography (CT) scan of patient with osteosarcoma of the ninth rib showing the extent of the tumor mass (upper left panel). Postoperative follow-up CT scan after thoracic wall resection and reconstruction after 6 months (upper right panel) and after 12 months (lower panel).

scans were used to evaluate the patches for structural changes and integrity.

\section{Histopathology and Immunohistochemistry}

Formalin fixated and paraffin embedded sections of the explanted patch material were stained for hematoxylin and eosin and periodic acid Schiff reaction. Immunohistochemistry (IHC) was used to stain for CD31 positive capillary endothelial cells indicating neoangiogenesis. IHC was performed by means of a standardized avidin-biotin complex method.

\section{Results}

Resection of chest wall sarcomas, including complete removal of the sternum in two cases, was successfully performed in all patients. Details of patient data and surgical procedures are listed in -Tables 1 and 2. A minimal area of three ribs with adjacent soft tissues was resected. Mean size of the defects was $149 \mathrm{~cm}^{2}$ (range: $80-252 \mathrm{~cm}^{2}$ ). Complete resection (R0) and wide safety margins were confirmed by histopathological examination in all patients.

The collagen patch material proved to be implantable under tension. The material was pliable but not stretchable, and resulted in immediate stability of the thoracic wall.

Mobilized subcutaneous tissue and skin were sufficient to cover the patches in four patients. Pediculate muscle flaps were necessary for two patients (Musculus latissimus dorsi and bilateral M. pectoralis major flap). Primary skin closure was achieved in all patients. Mean duration of surgery was 178 (range: 110-305) minutes.

All patients were extubated in the operating room and transferred to the intensive care unit $(n=3)$ or surgical ward $(n=3)$. The postoperative course was uncomplicated in all cases. In particular, no early or late respiratory impairment was observed. No postoperative bleeding, no infections, or wound healing problems were encountered. Inflammatory markers peaked in average (C-reactive protein [CRP] $29.2 \mathrm{mg} /$ $\mathrm{dL}$; white blood cell count [WBC] $12,950 / \mu \mathrm{L}$ ) on postoperative day 2. Mean CRP value at 3 weeks postoperatively was 1.2 (range: $0.5-4) \mathrm{mg} / \mathrm{dL}$ and mean $\mathrm{WBC}$ was 7,270 (range: 4,310-11,500)/ $\mu \mathrm{L}$. Chest drains were removed after a mean of 5 (range: 2-8) days. Mean hospital stay was 10 (range: 815 ) days. The 30 -day mortality was $0 \%$.

Mean duration of follow-up was 27.6 (range 8-42) months. None of the patients suffered from subjective respiratory limitations or complaints. No fluid accumulation inside or outside the thorax, no excessive fibrous tissue formation, or signs of herniation were observed (-Table 2 ).

Following lateral chest wall resections, provoking tests caused only minimal movements of the patch area. At 3month follow-up, the stability was rated as good $(n=2)$ and excellent $(n=2)$.

Initially, both cases of complete sternal reconstruction presented with noteworthy visible respiratory movements of the anterior chest. Neither of the patients suffered from respiratory impairment. In the course of follow-up, the stability improved significantly. At 3-month follow-up, the stability was rated good $(n=1)$ and moderate $(n=1)$ by the surgeon under deep breath and coughing tests ( - Table 2 ). In all six patients, no change in stability was observed at follow-up visits beyond 3 months. On routine follow-up CT scans, all implanted patches were identified as intact without bulging, herniation, rupture, or loss of structural integrity. The appearance of the patch material in the CT scans did not change during follow-up. No excessive encapsulation or seroma formations were evident. A representative series of follow-up CT scans demonstrating the intact patch material is shown in - Fig. $\mathbf{3}$. 


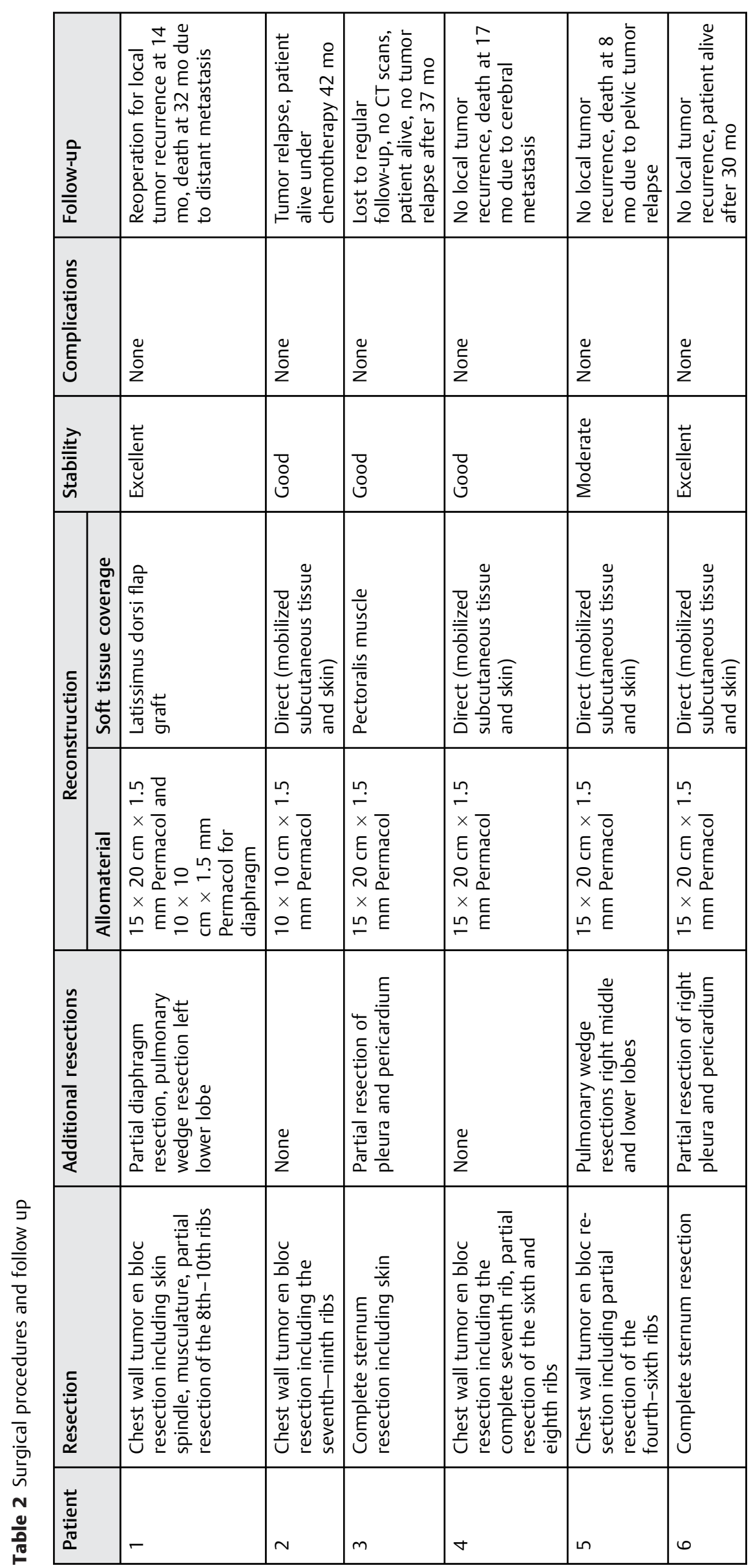



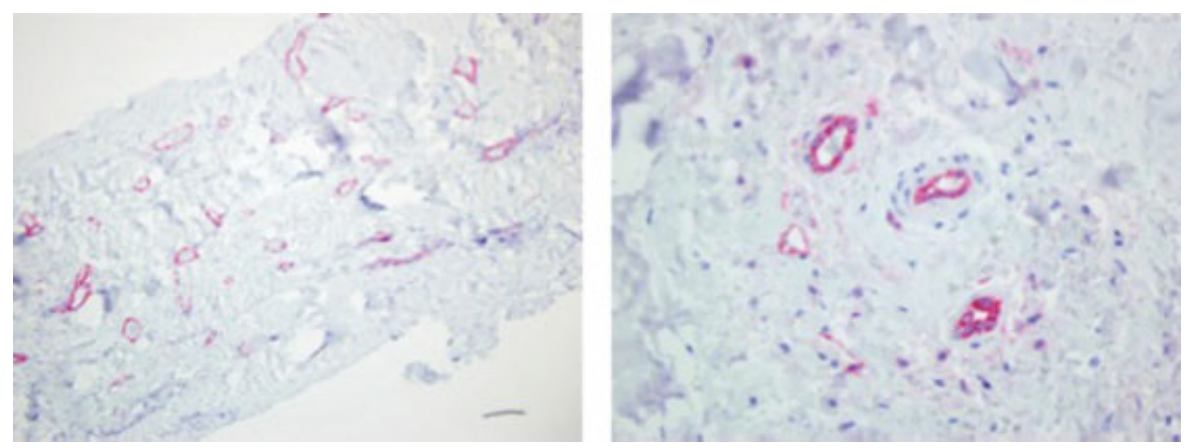

Fig. 4 Hematoxylin and eosin stain with immunohistochemistry of CD31 for neovascularization in explanted collagen matrix patch 14 months after initial implantation. Intimal cells are stained red. Tenfold magnification on left panel and 40-fold on right panel.

Follow-up and oncological outcome are shown in - Table 2. Despite wide safety margins, patient 1 developed a local tumor relapse and underwent reoperation 14 months after his first tumor resection. The primary implanted patch was removed en bloc with the recurrent sarcoma. During gross and histological examination, the patch proved to be intact and well integrated into the surrounding tissues (-Fig. 4). IHC staining for CD31 showed remarkable neovascularization of the collagen matrix within the surrounding tissue. This observation is a substantial indication for biological integration of the collagen matrix patch.

Upon returning to his mid-eastern country of residence 4 months postoperatively, patient 3 was lost to personal follow-up. According to e-mail contact, the patient is healthy, without local impairments and is free of tumor recurrence.

\section{Discussion}

Following its introduction nearly 30 years ago, PTFE has emerged as the most frequently applied material for chest wall reconstructions. It allows for implantation under tension and results in a durable replacement of bony and soft tissues. Some surgeons even used methylmethacrylate sandwich techniques or large metal prostheses to create sufficient stability in large defects, especially in cases of sternal resections. ${ }^{2-6,17}$ Nevertheless, reconstructions with synthetic materials are associated with serious complications. Synthetic materials are known to cause seroma formation and excessive encapsulation by dense fibrous tissue. The latter may not only result in patient discomfort and impaired respiration but also in serious deformities such as scoliosis following chest wall replacement in children. ${ }^{15,16}$

More importantly, PTFE materials do not overcome the main risk of all synthetic durable implants: infection. Although low complication rates have been described in some series, infection is a common problem, particularly in large reconstructions and sternal replacements ranging from 5 to $12 \%{ }^{2-6,8,9}$ Therefore, effective soft-tissue coverage with muscle flaps is essential, when applying synthetic allomaterials. A considerable number of synthetic patches had to be revised or explanted for these reasons. Mansour et al report 7\% of flap complications or loss, and another $5 \%$ of surgical site infection. ${ }^{4}$ In 2006, Weyant et al showed that within 30 days complication rates were $38 \%$ for rigid methylmethacrylate sandwich techniques and a 4.5\% 90-day prosthesis removal rate was observed. For PTFE or polypropylene mesh, the 30day mortality was $27 \%$ and the 90 -day prosthesis removal rate was $4.1 \%{ }^{6}$ Supposedly, absorbable or composite meshes have a reduced infection risk. However, these implants may result in significant mid- and long-term instabilities, paradoxical respiratory movements, and finally herniation in the area of the reconstructed chest wall. $1,3,4,8$

The rationale for employing collagen matrix patches in thoracic wall reconstruction is to combine the rigidity and durability of nonabsorbable synthetic materials with a reduced risk of infection. ${ }^{15,19}$

A growing variety of biological collagen matrix meshes is commercially available. Unlike permanently fixated biological materials, this novel generation of materials allows for a remodeling process with neovascularization and complete integration. This process starts with the migration of host cells and the formation of new blood vessels. In this respect, our histological findings (-Fig. 4) in a patch explanted after 14 months support the data from other reports. ${ }^{7,19}$

Our positive experiences with respect to strength, durability, and resistance to infection with this type of biological implant for thoracic wall reconstruction are in accordance with experimental and clinical findings of others. ${ }^{15-17,20}$ Most of the available data stem from the field of abdominal hernia repair. ${ }^{7,10,14,19}$ Only very few reports describe the application of collagen matrix patches in thoracic surgery. ${ }^{15-17,20}$ In abdominal hernia surgery, biological materials proved to be superior even to synthetic meshes with an overall success rate of $90 \% .{ }^{21}$ However, to our knowledge, no experimental study exists which compares the biomechanical characteristics of collagen matrixes and synthetic materials directly. In an animal study of hernia repair contaminated by staphylococci, all cases with synthetic implants failed, whereas $67 \%$ of the animals with the biological patch type used by us experienced uncomplicated wound healing. ${ }^{22}$ Clinical studies in patients with contamination and overt infection of the surgical field resulted in similar outcomes. ${ }^{23,24}$

However, for reconstruction of the thoracic wall characteristics of ideal materials and techniques of implantation are different. To minimize paradox respiratory movements, a patch must be implanted under tension and should not be stretchable. The ideal characteristics and strength of the biological material should resemble that of 1 - to 2-mm-thick 
PTFE membranes. Compared with other collagen matrixes, the product used in this study provides very good strength and durability owing to a thickness of $1.5 \mathrm{~mm}$.

At this point, we would like to stress that origin and processing of a collagen matrix determines remodeling, stability, and durability. Whereas reduced susceptibility to infection is a common attribute of all these implants, strength and durability are not.

Tissues derived from dermis, intestinal submucosa, or pericardium of porcine, bovine, and human origin have been explored. The process of chemical cross-linking restricts the extent of degradation by collagenase digestion and the turnover of collagen fibers following implantation. On the contrary, cellular infiltration, neovascularization, and integration are delayed as well. ${ }^{7,17}$ Compared with a collagen matrix derived from porcine dermis, significantly higher failure rates were reported for non-cross-linked human acellular dermis in a systematic review of the published series of hernia repair. Non-cross-linked collagen mesh from human dermis, which undergoes extensive remodeling and is known to stretch with time, seems to be less suitable for use in thoracic surgery. ${ }^{25}$

Application of collagen matrixes may also be beneficial for pediatric patients. Deformities of the spine are a well-known complication following thoracic wall reconstruction in children. ${ }^{15}$ Lin et al demonstrated that chest wall reconstructions with collagen matrix patches in pediatric patients did not lead to the development of clinically relevant scoliosis.

Handling and tailoring of the thick collagen patch was easy, it proved to be blood tight and a safe abutment for sutures. No seroma formation, wound healing problems, or infections were observed in this pilot study. The clinical, radiological, and histological findings in this study support long-term stability and effective integration of this type of collagen matrix implant. Our experience demonstrates that a porcine dermal-derived acellular collagen matrix is a viable alternative to PTFE for thoracic wall reconstructions. However, it is evident that this pilot study only allows limited conclusions. Also, the advantages of collagen matrix materials must be weighed against the considerably higher expense. Nevertheless, in complicated settings such as large defects, total sternal replacement, difficult soft-tissue coverage, contaminated surgical field, or pediatric surgeries, implantation of this biological patch appears to be justified.

\section{Disclaimer}

The German Society for Thoracic and Cardiovascular Surgery (DGTHG) and The Thoracic and Cardiovascular Surgeon neither endorse nor discourage the use of the new technology described in this publication.

\section{Conflict of Interest}

None of the authors has a conflict of interest to disclose.

\section{References}

1 Mahabir RC, Butler CE. Stabilization of the chest wall: autologous and alloplastic reconstructions. Semin Plast Surg 2011;25(1):34-42

2 Smith SE, Keshavjee S. Primary chest wall tumors. Thorac Surg Clin 2010;20(4):495-507

3 Arnold PG, Pairolero PC. Chest-wall reconstruction: an account of 500 consecutive patients. Plast Reconstr Surg 1996;98(5):804-810
4 Mansour KA, Thourani VH, Losken A, et al. Chest wall resections and reconstruction: a 25-year experience. Ann Thorac Surg 2002; 73(6):1720-1725, discussion 1725-1726

5 Deschamps C, Tirnaksiz BM, Darbandi R, et al. Early and long-term results of prosthetic chest wall reconstruction. J Thorac Cardiovasc Surg 1999;117(3):588-591, discussion 591-592

6 Weyant MJ, Bains MS, Venkatraman E, et al. Results of chest wall resection and reconstruction with and without rigid prosthesis. Ann Thorac Surg 2006;81(1):279-285

7 Holton LH III, Chung T, Silverman RP, et al. Comparison of acellular dermal matrix and synthetic mesh for lateral chest wall reconstruction in a rabbit model. Plast Reconstr Surg 2007;119(4):1238-1246

8 Nagayasu T, Yamasaki N, Tagawa T, et al. Long-term results of chest wall reconstruction with DualMesh. Interact Cardiovasc Thorac Surg 2010;11(5):581-584

9 Rocco G. Overview on current and future materials for chest wall reconstruction. Thorac Surg Clin 2010;20(4):559-562

10 Deeken CR, Melman L, Jenkins ED, Greco SC, Frisella MM, Matthews BD. Histologic and biomechanical evaluation of crosslinked and non-crosslinked biologic meshes in a porcine model of ventral incisional hernia repair. J Am Coll Surg 2011;212(5):880-888

11 Adedeji OA, Bailey CA, Varma JS. Porcine dermal collagen graft in abdominal-wall reconstruction. Br J Plast Surg 2002;55(1):85-86

12 Harper C. Permacol: clinical experience with a new biomaterial. Hosp Med 2001;62(2):90-95

13 Miller DL, Force SD, Pickens A, Fernandez FG, Luu T, Mansour KA. Chest wall reconstruction using biomaterials. Ann Thorac Surg 2013;95(3):1050-1056

14 Hsu PW, Salgado CJ, Kent K, et al. Evaluation of porcine dermal collagen (Permacol) used in abdominal wall reconstruction. J Plast Reconstr Aesthet Surg 2009;62(11):1484-1489

15 Lin SR, Kastenberg ZJ, Bruzoni M, Albanese CT, Dutta S. Chest wall reconstruction using implantable cross-linked porcine dermal collagen matrix (Permacol). J Pediatr Surg 2012;47(7):1472-1475

16 Mirzabeigi MN, Moore JH Jr, Tuma GA. The use of Permacol® for chest wall reconstruction in a case of desmoid tumour resection. J Plast Reconstr Aesthet Surg 2011;64(3):406-408

17 Rocco G, Mori S, Fazioli F, La Rocca A, Martucci N, Setola S. The use of biomaterials for chest wall reconstruction 30 years after radical surgery and radiation. Ann Thorac Surg 2012;94(4):e109-e110

18 Melman L, Jenkins ED, Hamilton NA, et al. Early biocompatibility of crosslinked and non-crosslinked biologic meshes in a porcine model of ventral hernia repair. Hernia 2011;15(2):157-164

19 Deeken CR, Abdo MS, Frisella MM, Matthews BD. Physicomechanical evaluation of polypropylene, polyester, and polytetrafluoroethylene meshes for inguinal hernia repair. J Am Coll Surg 2011; 212(1):68-79

20 Murphy F, Corbally MT. The novel use of small intestinal submucosal matrix for chest wall reconstruction following Ewing's tumour resection. Pediatr Surg Int 2007;23(4):353-356

21 Hiles M, Record Ritchie RD, Altizer AM. Are biologic grafts effective for hernia repair?: a systematic review of the literature Surg Innov 2009;16(1):26-37

22 Harth KC, Broome AM, Jacobs MR, et al. Bacterial clearance of biologic grafts used in hernia repair: an experimental study. Surg Endosc 2011;25(7):2224-2229

23 Butler CE, Langstein HN, Kronowitz SJ. Pelvic, abdominal, and chest wall reconstruction with AlloDerm in patients at increased risk for mesh-related complications. Plast Reconstr Surg 2005;116(5): 1263-1275, discussion 1276-1277

24 Rosen MJ, Krpata DM, Ermlich B, Blatnik JA. A 5-year clinical experience with single-staged repairs of infected and contaminated abdominal wall defects utilizing biologic mesh. Ann Surg 2013; 257(6):991-996

25 Jansen LA, De Caigny P, Guay NA, Lineaweaver WC, Shokrollahi K. The evidence base for the acellular dermal matrix AlloDerm: a systematic review. Ann Plast Surg 2013;70(5):587-594 